Sensors Applications

\title{
Network Optimization for Industrial Internet of Things (IloT)
}

\author{
Ruhul Amin Khalil $^{1 *}$ and Nasir Saeed ${ }^{2 * *}$ \\ ${ }^{1}$ Department of Electrical Engineering, Faculty of Electrical and Computer Engineering, University of Engineering and Technology, \\ Peshawar 25120, Pakistan \\ ${ }^{2}$ Computer, Electrical, and Mathematical Sciences \& Engineering (CEMSE) Division King Abdullah University of Science and Technology, \\ Thuwal 23955, Makkah, Kingdom of Saudi Arabia \\ * Student Member, IEEE \\ ** Senior Member, IEEE \\ Manuscript received .., 2020; revised $\cdots$, 2020; accepted $\cdots, 2020$.
}

Abstract-Industrial Internet of Things (IloT) requires reliable, low-latency and secure wireless links to support rate/delay/security-intensive applications. In this letter, we model the IloT network as a geometric graph with a virtual coordinate system (VCS). One major problem with VCS is that it is sensitive to the placement of reference nodes. This letter first proposes a general formulation for selecting a specific set of reference nodes that minimizes the network error. Then, we derive the upper bound for the optimization problem and present a novel iterative method that selects additional reference nodes based on the network inference. Simulation results compare the proposed method with the random selection of reference nodes and show that the proposed method performs better regarding packet delivery rate, network connectivity, and total energy consumption.

Index Terms—-Industrial Internet of Things, graph, optimization, virtual coordinate system

\section{INTRODUCTION}

Industrial Internet of Things (IIoT) is a network of interconnected sensors and instruments that enables smart and adaptable manufacturing in the forthcoming industrial 4.0 concept. IIoT utilizes modern sensor technology for interconnecting and digitalizing traditional factories [1]-[3]. This can facilitate self-organizing, remote monitoring, and collaborative control for the conventional industries. The basic framework of IIoT consists of sensors, actuators, and edge nodes connected to the gateway as shown in Fig. 1. The gateway is further connected to the IoT platform where the data analysis and processing are achieved. The primary reason for establishing IIoT is that intelligent machines efficiently collect, communicate, and act in real-time industrial applications [4]. To achieve this, IIoT first requires stable and uninterrupted high data-rate communication to support real-time monitoring, data collection, and applications of cloud/edge computing. Also, it demands wide-area coverage, massiveand-ubiquitous connectivity, low-latency below one millisecond, and ultra-reliability with packet loss rate below $10^{-12}$ [5]. Moreover, manufacturing related information leakage may cause core secret disclosures and malicious attacks, which may ultimately paralyze the entire production process. Therefore, communication security is also of paramount importance in the IIoT [6].

Consequently, to meet all of the above requirements in IIoT, both industry and academia have been urged to develop new wireless communication technologies for the industrial environments. For instance, in [5], the authors proposed a 5G-enabled IIoT architecture, which shows that how the key potentials of 5G, such as ultrareliable and low-latency communication (URLLC), enhanced-mobilebroadband (eMBB), and massive machine-type communication (mMTC) can help in automation of the industries. The authors of [7] proposed a load balancing scheme that dynamically manages the

Corresponding author: Nasir Saeed (e-mail: mr.nasir.saeed@ieee.org). (https://cemse.kaust.edu.sa/ctl/people/person/nasir-saeed). Digital Object Identifier 10.1109/LSENS.2020.0000000

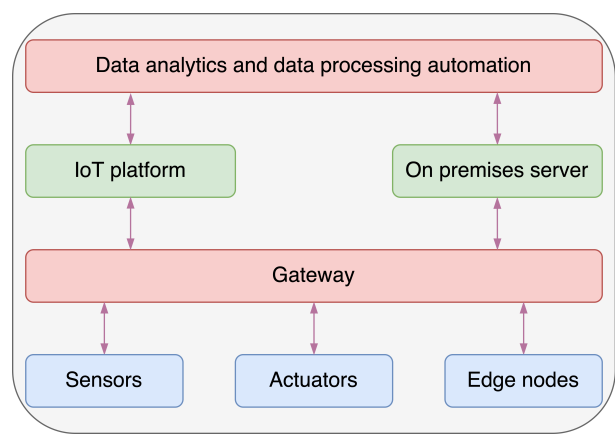

Fig. 1: Basic framework of Industrial IoT.

wireless links according to the link quality and data load. Moreover, the authors in [8] discussed the use of millimeter waves and low Terahertz frequency band for IIoT systems.

Besides the communication issue, topology optimization in IIoT is of great importance. Based on the coordinates of the nodes, there are mainly two localization systems, geographical coordinate system (GPS) and virtual coordinate system (VCS). GPS method uses actual coordinates of nodes and therefore is more costly and complicated. Also, GPS is vulnerable to channel impairments, ranging error, and multi-path fading. On the contrary, VCS is more simple and easy to implement since it only depends on the hop measurements [9], [10]. The VCS first selects the reference nodes, and then each reference node measures the total number of hops separating it from the remaining nodes. Each node in this method follows an incrementand-forward approach concerning its close-by nodes [11]. Hence, this simple procedure makes VCS robust to the noise and channel distortion since it considers only the connectivity.

One primary issue with the VCS method is that if the reference nodes are not selected optimally, the generated VCS will suffer from identical coordinates and local minima problems [12]. Placement and selection of reference nodes are critical because the proximity map generated in VCS can result in significant network error in terms of connectivity if the reference nodes are not optimally placed 
or selected. To elaborate further, for example, in multidimensional scaling-based VCS, if the selected reference nodes are co-linear, it results in a flipping ambiguity that further introduces a large connectivity error in the network [13]. Not only that, but such a network ambiguity also severely affects the routing performance of the system. All of the works mentioned above select the reference nodes randomly, which can result in a significant error. To the best of our knowledge, this work is the first attempt to investigate the problem of reference node selection for network optimization in IIoT. Few recent attempts proposed a reference node placement solution [14] and outlier detection [15]. However, none of them tackle the issue of reference node selection to get accurate results. Therefore, in this letter, we propose a reference node selection method that improves network connectivity and accurate VCS. First, we formulate the reference node selection problem regarding the network inference error. Then, we derive the optimization problem and its upper bound. Finally, we propose an iterative reference node selection method that provides a near-optimal solution.

This letter is organized as follows. Section II introduces the system model and presents the formulation of the reference node selection problem. In Section III, we show the proposed method for solving the optimization problem. Section IV provides the numerical results that demonstrate the advantages of our proposed scheme compared to the literature. Finally, Section V concludes the letter with a few remarks.

\section{PROPOSED SYSTEM MODEL}

We consider an industrial IoT network consisting of $N$ number of nodes as shown in Fig. 2. The nodes are represented by set of vertices $\mathcal{N}=\left\{n_{1}, n_{2}, \cdots, n_{N}\right\}$ and corresponding set of edges $E \subset \mathcal{N} \times \mathcal{N}$. Then, the connectivity matrix $\mathbf{C}$ for such a topology is a $N \times N$ symmetric matrix, where the edge between any two nodes $i$ and $j$ is represented by $c_{i j}$. If the two nodes are connected then $c_{i, j}=1$ otherwise $c_{i, j}=0$. Also, we assume that the network is connected which means that there is always a path between any pair of nodes. Consider that $\mathcal{E}$ is the space of all possible connectivity matrices $\mathbf{C}$ for such a graph. Since $c(i, j)=c(j, i)$ and $c(i, i)=0$, it is clear that $\mathcal{E} \subset\{0,1\}^{\frac{N(N-1)}{2}}$.

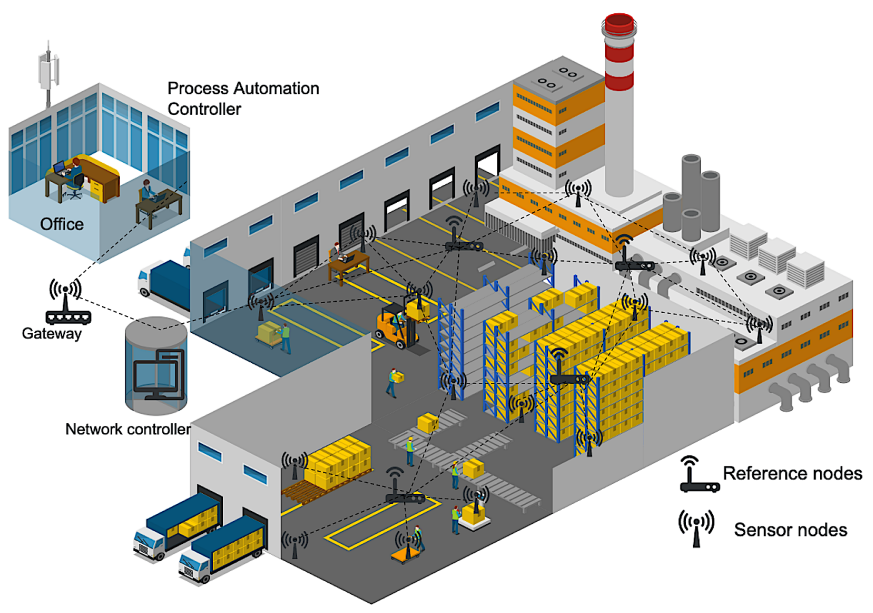

Fig. 2: Network architecture for Industrial IoT.

We consider $M<N$ reference nodes with connectivity matrix $\mathcal{R}=\left\{R_{1}, R_{2}, \cdots, R_{M}\right\} \subset \mathcal{N}$. These reference nodes are used to collect the hop measurements from ordinary nodes using controlled network flooding. This process starts when the reference nodes transmit the probing packets to the remaining nodes in the network. In response, each node calculates the number of hops to the reference nodes and forwards the shortest path (minimum number of hopes to the reference nodes) to the reference nodes. When the flooding process completes, the reference nodes collect all of the hop distance measurements from the ordinary nodes and create a distance matrix $\mathbf{H}(\mathcal{R})$. Note that the $(i, j)^{t h}$ entry of $\mathbf{H}(\mathcal{R})$ represents the shortest hop distance between ordinary node $n_{i}$ and reference node $R_{j}$. As each ordinary node $n_{i}$ creates a vector $\mathbf{h}_{i}$ consisting of hop distances to the $M$ reference nodes, we can write the hop measurements matrix relative to the set of reference nodes $\mathcal{R}$ as

$$
\mathbf{H}(\mathcal{R})=\left[\mathbf{h}_{1}^{T}, \mathbf{h}_{2}^{T}, \cdots, \mathbf{h}_{N}^{T}\right],
$$

where $\mathbf{h}_{i}$ represents the virtual coordinate vector for node $n_{i}$. Now using this virtual coordinate system (VCS), we define the logical distance $d_{\mathcal{R}}$ relative to the reference nodes set $\mathcal{R}$ :

$$
d_{\mathcal{R}}\left(n_{i}, n_{j}\right)=\left\|\mathbf{h}_{i}-\mathbf{h}_{j}\right\|_{2} .
$$

Further, we formulate the joint problem of reference node selection and network optimization. Consider that $P\left(e_{k} \mid \mathcal{R}\right)$ is the probability that $e_{k} \in \mathcal{E}$ is the solution of the adjacency matrix for a given $\mathbf{H}(\mathcal{R})$. Also, $\hat{e}_{k}$ is the estimated adjacency matrix, then the probability of error in adjacency matrix is given as:

$$
P_{e}(\mathcal{R})=\sum_{e_{k} \in \mathcal{E}} \sum_{j \neq k} P\left(e_{k} \mid \mathcal{R}\right) P\left(\hat{e}_{k}=e_{j} \mid e_{k} ; \mathcal{R}\right) .
$$

Additionally, the problem of selecting optimal reference node set $\mathcal{R}^{*}$ can be formulated as:

$$
\begin{gathered}
\min _{\mathcal{R}} P_{e}(\mathcal{R}) \\
\text { s.t. }|\mathcal{R}| \leq M .
\end{gathered}
$$

In the next section we elaborate this optimization problem and propose an iterative approach to find a near-optimal solution.

\section{PROPOSED REFERENCE NODE SELECTION TECHNIQUE}

In this section, we develop a reference node selection scheme for network connectivity performance that provides efficient and reliable VCS. Assuming that the hop measurements from all the nodes are known, then the problem defined in (4) is NP-hard, and therefore, it requires exponential running time [16]. However, in practice, the computational power is limited, and only a set of hop measurements to the reference nodes is available. Hence, we relax both these assumptions and solve the problem iteratively where at each iteration, the connectivity matrix is updated along with the selection of the reference nodes. Let $\mathcal{R}^{(i)}$ is the set of reference nodes at the $i$ th iteration. Also, we assume that there is no prior information regarding the network topology; therefore, we initialize $\mathcal{R}^{(1)}$ randomly. Then, we increment the set of reference nodes at the $i$ th iteration such that the probability of error $P_{e}(\cdot)$ is minimized as follows:

$$
n^{(i)}=\underset{n \in \mathcal{N} \mid \mathcal{R}^{(i-1)}}{\arg \min } P_{e}\left(\mathcal{R}^{(i-1)} \cup n\right),
$$

where $n^{(i)}$ is the optimal set of nodes.

Unfortunately, estimating $P_{e}(\cdot)$ is hard due to the practical constraint of limited number of hop measurements. Therefore, we relax this constraint by defining an upper bound that is derived in each iteration by using the hop measurements. We derive the upper bound using 
the network optimization approach by relating $\mathbf{H}(\mathcal{R})$ and $\mathbf{C}$. This idea consists of the following steps:

- First, we organize the nodes into layers where the connectivity among any two arbitrary nodes $n_{i}$ and $n_{j}$ is defined as connected, disconnected, and ambiguous based on the following criteria:

- Nodes $n_{i}$ and $n_{j}$ are connected, if their hop distances are exactly one hope with respect to the same reference node.

- Nodes $n_{i}$ and $n_{j}$ are disconnected, if their hop distances are greater than one hop with respect to same reference node.

- The connection between nodes $n_{i}$ and $n_{j}$ is ambiguous, if there is no possibility to determine the connectivity information. The ambiguity arises due to flipping issue in VCS [17].

- This classification results in a partially filled connectivity matrix with a certain ambiguity. We consider $\alpha(\mathcal{R})$ as the ambiguity in the connectivity matrix.

- Then using the fact that probability of error is upper bounded by $P_{e}(\mathcal{R})<\alpha(\mathcal{R})$. Using this bound, the problem of reference node selection is relaxed as follows:

$$
n^{(i)}=\underset{n \in \mathcal{N} \mid \mathcal{R}^{(i-1)}}{\arg \min } \alpha\left(\mathcal{R}^{(i-1)} \cup n\right) .
$$

This proposed iterative method is summarized in Algorithm-1 by starting with a selection of random set of reference nodes. Then, based on (6), we select reference nodes based on their connectivity ambiguity function. Nevertheless, it is still challenging to solve (6)

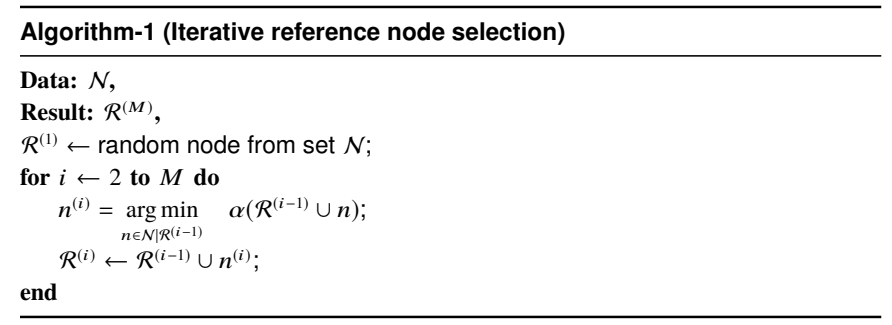

because it needs the connectivity information relative to the nodes in $\mathcal{N} \mid \mathcal{R}^{(i-1)}$ which is not accessible at the $i$ th iteration. To solve this issue, we use a greedy approach to estimate $\alpha\left(\mathcal{R}^{(i)}\right)$ using only the available connectivity information from the set of reference nodes $\mathcal{R}^{(i-1)}$. Moreover, the reference node selection strategy at the $i$ th iteration consists of the following steps:

- First, we select the farthest node in terms of the logical distance $d_{\mathcal{R}^{(i-1)}}(\cdot)$, of the current set of reference nodes $\mathcal{R}^{(i-1)}$. Intuitively, this criterion selects a reference node that is far from the current set of reference nodes to explore network depth, i.e.,

$$
n^{(i)}=\underset{n \in \mathcal{N} \mid \mathcal{R}^{(i-1)}}{\arg \min }\left(\min _{m \in \mathcal{R}^{(i-1)}} d_{\mathcal{R}^{(i-1)}}(n, m)\right) .
$$

- Then we choose the node that has a maximum number of ambiguous edges based on the connectivity matrix with respect to the set of reference nodes $\mathcal{R}^{(i-1)}$. This criterion minimizes the local ambiguity, i.e.,

$$
n^{(i)}=\underset{n \in \mathcal{N} \mid \mathcal{R}^{(i-1)}}{\arg \min }\left(\alpha^{(i-1)}(n)\right),
$$

where $\alpha^{(i-1)}(n)$ is the local ambiguity of node $n$ at the $(i-1)$ th iteration.

- Finally, our proposed criterion state that if we obtain multiple entries from the second criterion, we use the first criterion to decide. It means that if there is possibility of multiple candidates with equal ambiguity function, then we decide based on the first criteria, i.e., based on the logical distance.

\section{RESULTS AND DISCUSSIONS}

In this section, we test our proposed reference node selection method using MATLAB regarding the connectivity error, packet delivery rate, and total energy consumption. The simulation parameters used throughout this section are given in Table. 1. First, we generate a

Table 1: Parameters used for simulations

\begin{tabular}{ll}
\hline Parameter & Value \\
\hline Simulation Area & $100 \mathrm{~m} \times 100 \mathrm{~m}$ \\
Communication range & $50 \mathrm{~m}$ \\
Number of reference nodes $\mathcal{R}$ & 0 to 30 \\
Number of sensor nodes $N$ & 100 \\
Simulation runs & 500 \\
Percentage of non-zero entries $\beta$ & $15 \%$ \\
Sensitivity threshold & $-120 \mathrm{~dB}$ \\
\hline
\end{tabular}

random industrial IoT network with $N$ number of sensor nodes in a two-dimensional area. Next, we assign edges randomly between pairs of sensor nodes such that the network graph is connected. Consider that $\beta=|E|(N(N-1)) / 2$ represents the percentage of non-zero entries in the connectivity matrix $\mathbf{C}$. Then, we select a subset of reference nodes based on the different criteria discussed in Section-III. Finally, we use the controlled flooding method to collect the hop measurements from the reference nodes. Also, we evaluate the performance of the proposed method regarding total energy consumption since it is an essential parameter for batterypowered IoT nodes. We compare the total energy consumption of the proposed method and the random reference selection method. Note that the results are averaged over 500 Monte Carlo simulations. Also, we vary the number of reference nodes while fixing $N=100$ and $\beta=15 \%$ (minimum threshold for a connected network).

\section{A. Connectivity Ambiguity}

Fig. 3a compares the performance of various reference node selection techniques regarding network connectivity ambiguity and the number of reference nodes. Also, we examine the results with the optimal solution of (5) and exhaustive search of (4). Fig. 3a shows that our proposed method nearly follows the optimal solution. However, the exhaustive search has slightly better performance when the number of reference nodes is small due to unavailable connectivity information. Indeed, as mentioned in Algorithm-1, the first reference node $\mathcal{R}^{(1)}$ is randomly selected.

\section{B. Packet Delivery Rate}

Next, we investigate the routing performance of the proposed scheme by randomly selecting a source and destination node. Also, we compare the results of logical-distance-based routing (LDR) with random reference nodes and with the reference node selection strategy. LDR is inspired partly by the classical distance vector routing technique and is based on the measurements of number hop counts between the reference nodes. It simply forwards the data packets towards the neighboring nodes that are closest to the destination nodes relative in terms of logical distance. LDR has few shortcomings that include the risk of infinite looping and local 


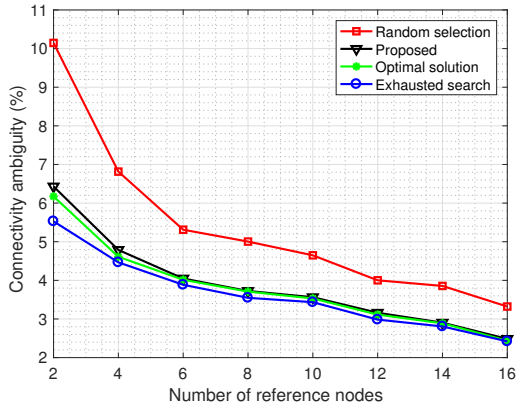

(a)

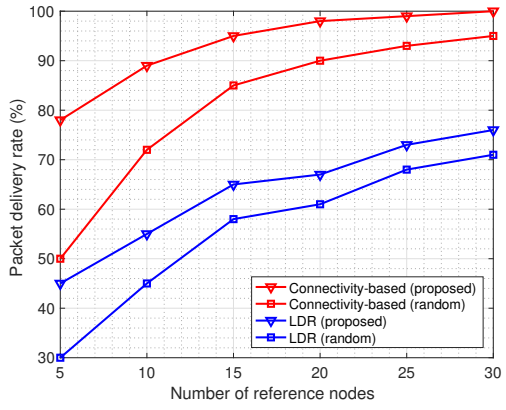

(b)

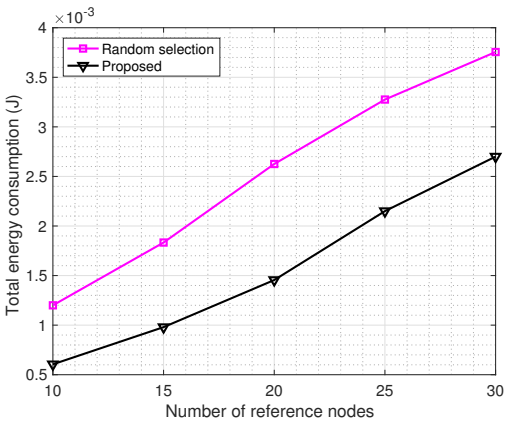

(c)

Fig. 3: (a) Number of reference nodes Vs. Connectivity ambiguity; (b) Number of reference nodes Vs. Packet delivery rate; (c) Number of reference nodes Vs. Total energy consumption.

minima that occurs due to the ambiguity of virtual coordinates. On the other hand, the connectivity-based method calculates the shortest path between the source and the destination node, and if there is no connectivity ambiguity, the shortest path can be perfectly calculated by the well-known Dijkstra algorithm. In the current scenario, the connectivity ambiguity can cause packet delivery loss due to the presence of error in the connectivity-based matrix. Moreover, the packet delivery rate improves with the increase in the number of reference nodes for both the cases. Note that for the packet delivery rate, we consider the percentage of successfully delivered packets to the destination. Fig. $3 b$ shows the delivery-rate for the connectivitybased and LDR approach. It is clear from Fig. 3b that the reference node selection improves the packet delivery-rate by $20 \%$. Also, the proposed method outperforms the LDR due to the reference node selection strategy.

\section{Total Energy Consumption}

Also, we show the performance of the proposed scheme in terms of total energy consumption with an increase in the number of reference nodes. The total energy $E_{T}$ consists of basic electronic operation, transmission energy and residual energy. The total energy consumed by all nodes $(M+N)$ in the IIoT network is calculated as

$$
E_{T}=\sum_{i=1}^{M+N} E_{e_{i}}+(M+N)\left(\sum_{i=1}^{M+N} E_{t_{i}}+\sum_{i=1}^{M+N} E_{r_{i}}\right),
$$

where $E_{e_{i}}$ is the energy consumed for basic electronics operation, $E_{t_{i}}$ represents the transmission energy, and $E_{r_{i}}$ is the residual energy of $i$-th node, respectively. Fig. $3 \mathrm{c}$ shows that the overall energy consumption of the network increases as the number of reference nodes increases. Furthermore, we compare the results with connectivity-based routing with random reference nodes selection criteria. Fig. $3 \mathrm{c}$ illustrates that the proposed scheme uses less energy because it uses local information only from the neighboring nodes and does not coordinate with the reference nodes beyond their hope range, thus reducing the total energy consumption.

\section{CONCLUSION}

In this letter, we proposed a novel reference node selection method and evaluated its routing performance for industrial Internet of Things (IIoT). First, we modeled the IIoT system as a theoretical graph to capture the underlying structure. Then, we utilized the ambiguity function to exploit the connectivity information for estimating the probability of network error. Further, we proposed an iterative method to determine the optimal set of reference nodes based on specific selection criteria. We show that our proposed method accurately generates a virtual coordinate system for the IIoT network. Moreover, our results show that the proposed method is nearly optimal, energy efficient and outperforms the random selection method, concerning the packet delivery rate and network connectivity.

\section{REFERENCES}

[1] A. Gilchrist, Industry 4.0: The industrial internet of things. Springer, 2016.

[2] H. Boyes, B. Hallaq, J. Cunningham, and T. Watson, "The industrial internet of things (IIoT): An analysis framework," Computers in Industry, vol. 101, pp. 1-12, 2018.

[3] J. Wan, S. Tang, Z. Shu, D. Li, S. Wang, M. Imran, and A. V. Vasilakos, "Softwaredefined industrial internet of things in the context of industry 4.0," IEEE Sensors J., vol. 16, no. 20, pp. 7373-7380, 2016.

[4] E. Sisinni, A. Saifullah, S. Han, U. Jennehag, and M. Gidlund, "Industrial internet of things: Challenges, opportunities, and directions," IEEE Trans. Ind. Informat., vol. 14, no. 11, pp. 4724-4734, 2018.

[5] J. Cheng, W. Chen, F. Tao, and C.-L. Lin, "Industrial IoT in 5G environment towards smart manufacturing," J. Ind. Inf. Integr., vol. 10, pp. 10-19, 2018.

[6] Z. Bi, L. Da Xu, and C. Wang, "Internet of things for enterprise systems of modern manufacturing," IEEE Trans. on Ind Informat., vol. 10, no. 2, pp. 1537-1546, 2014.

[7] M. C. Lucas-Estañ and J. Gozalvez, "Load balancing for reliable self-organizing industrial IoT networks," IEEE Trans. Ind. Informat., vol. 15, no. 9, pp. 5052-5063, 2019.

[8] T. Yilmaz and O. B. Akan, "On the use of the millimeter wave and low terahertz bands for internet of things," in IEEE WF-IoT, 2015, pp. 177-180.

[9] A. P. Jayasumana, R. Paffenroth, G. Mahindre, S. Ramasamy, and K. Gajamannage, "Network topology mapping from partial virtual coordinates and graph geodesics," IEEE/ACM Trans Net., vol. 27, no. 6, pp. 2405-2417, 2019.

[10] A. Caruso, S. Chessa, S. De, and A. Urpi, "GPS free coordinate assignment and routing in wireless sensor networks," in Proc. of 24th Annual Joint Conf. of the IEEE Comp. and Commun. Soc., vol. 1, 2005, pp. 150-160 vol. 1.

[11] Y. Zhao, Y. Chen, B. Li, and Q. Zhang, "Hop ID: A virtual coordinate based routing for sparse mobile ad hoc networks," IEEE Trans. Mobile Comput., vol. 6, no. 9, pp. 1075-1089, 2007.

[12] N. Saeed and H. Nam, "Robust multidimensional scaling for cognitive radio network localization," IEEE Trans. Veh. Technol., vol. 64, no. 9, pp. 4056-4062, 2015.

[13] N. Saeed, H. Nam, M. I. U. Haq, and D. B. Muhammad Saqib, "A survey on multidimensional scaling," ACM Comput. Surv., vol. 51, no. 3, pp. 1-25, 2018.

[14] N. Saeed, M. Alouini, and T. Y. Al-Naffouri, "Accurate 3-D localization of selected smart objects in optical internet of underwater things," IEEE Internet of Things J., vol. 7, no. 2, pp. 937-947, 2020.

[15] N. Saeed, T. Y. Al-Naffouri, and M.-S. Alouini, "Outlier detection and optimal anchor placement for 3-D underwater optical wireless sensor network localization," IEEE Trans. on Commun., vol. 67, no. 1, pp. 611-622, 2018.

[16] J. C. Lagarias and A. M. Odlyzko, "Solving low-density subset sum problems," J. ACM, vol. 32, no. 1, pp. 229-246, 1985.

[17] Z. Zhong and T. He, "Achieving range-free localization beyond connectivity," in Proc. of the 7th ACM Conf. on Emb. Netw. Sensor Sys., 2009, pp. 281-294. 